\title{
Tysabri withdrawal calls entire class into question
}

The future of a promising treatment for multiple sclerosis (MS) is in question after two patients takinag the drug were diagnosed with a rare neurological disorder.

Scientists are scrambling to determine the exact relationship between the drug, Tysabri, and progressive multifocal leukoencephalopathy (PML). If the drug is deemed to pose a significant risk, it could be a serious setback for the entire class of drugs, which work by blocking passage of immune cells into the brain.

"Other cell-migration inhibitors in development will have to be carefully tested," says Howard Weiner, director of the Partners MS Center at Brigham and Women's Hospital.

Several companies are developing drugs that use the same rationale to treat Crohn disease, rheumatoid arthritis and asthma. But according to Lawrence Steinman, an MS specialist at Stanford University, all of these drugs are likely to carry a similar risk for infections.
The US Food and Drug Administration (FDA) has halted a phase 2 trial of a GlaxoSmithKline drug that targets the same protein, alpha-4 integrin, as Tysabri. Australian biotech Antisense Therapeutics stopped phase 2 trials in March for a similar MS drug, which the company had also planned to develop as an asthma treatment. Switzerland-based Roche also has a similar compound in early clinical trials, but declined to comment on its plans for the trial.

Steinman has warned for more than a decade that such drugs might carry nasty side effects. In 1992, he was one of the first to develop this new approach — to block the homing molecules that allow immune cells to access the brain. But he says he soon realized that targeting these molecules also blocks immune cells from entering infected tissue outside the brain, leaving patients susceptible to infections. This might have led to the cases of PML, a disorder most often seen in people with compromised immune systems, such as those with AIDS, Steinman says.
The FDA in November 2004 granted Tysabri fast-track approval, after initial phase 3 results showed the drug reduces the frequency of MS attacks by as much as $66 \%$.

Tysabri was expected to be a major blockbuster for its manufacturer Biogen Idec, with predicted sales of $\$ 3$ billion annually. But the company suspended sales of Tysabri in February, after learning of the two PML cases, one of which was fatal. The company also halted trials of the drug for rheumatoid arthritis and Crohn disease.

There has never previously been a documented case of PML in MS patients, even though these patients have been treated with many types of immunosuppressants. Both patients diagnosed with PML had taken Tysabri with Avonex, another MS drug, for two years in clinical trials. Biogen is testing all patients who took Tysabri for signs of PML.

Emily Singer, Boston

\section{Experts inject reality}

Two rival vaccines to prevent cervical cancer are being launched with great fanfare as a long-awaited solution to a pressing public health problem. But distributing and administering the vaccines in developing countries, which bear much of the global burden of the infection, is likely to be costly and complicated, scientists warn.

Because the vaccine must be given to girls before they are sexually active, convincing parents to vaccinate prepubescent girls against a sexually transmitted infection will be fraught with cultural issues, experts say.

Cervical cancer is the second biggest cause of female cancer mortality worldwide, with 288,000 deaths annually. About 510,000 cases are reported each year, $80 \%$ of them in developing countries. India alone has onethird of the global burden.

Drug giants GlaxoSmithKline and Merck have both developed vaccines that target the human papilloma virus (HPV), which is linked to three-quarters of the infections. Both vaccines are in large phase 3 trials and are expected to reach the market in two years.

The vaccines carry the $\mathrm{L} 1$ capsid protein and need to be given in three doses to generate high levels of neutralizing antibodies. The GlaxoSmithKline vaccine is designed against the two most infectious HPV strains, HPV 16 and 18 , and the Merck vaccine against four strains-HPV 16, 18, 6 and 11. Both are safe and have shown efficacy of more than $90 \%$ in phase 1 and 2 trials.

But key scientific questions such as the duration and correlates of protection, the effect of the menstrual cycle and the need for a booster vaccine remain answered, cautions John Schiller, a researcher at the US National Cancer Institute.

The vaccines are also expensive to make and difficult to distribute, Schiller says. Administering three injections, maintaining clean, sterilized needles and creating a cold chain for transport to rural areas in resource-poor countries are all likely to pose significant hurdles, Schiller notes.

Developing countries may have to wait for a second-generation vaccine that is cheaper and easier to administer, protects against more HPV types and that better suits their needs. But "such a vaccine is not really on the horizon now," says Schiller. Alternately, they could make their own cheaper vaccine-which could be difficult with existing patent rights on the technology.

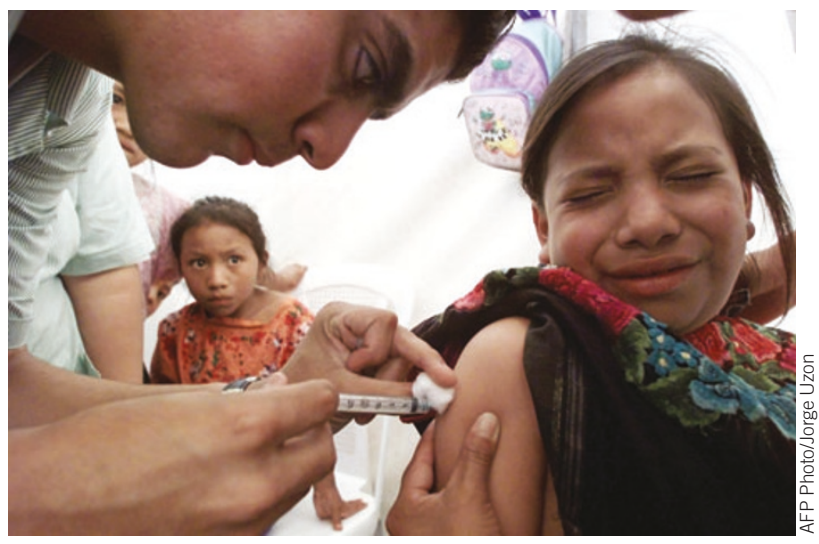

Culture shot: Parents in developing countries might be reluctant to vaccinate their young daughters against cervical cancer.

Another significant problem will be getting consent to vaccinate girls. For example, convincing parents in India-where sex education is taboo in most parts- to inject their daughters against a sexually transmitted infection will be impossible, says Bhudev Das, director of the Institute of Cytology and Preventive Oncology in Delhi.

Although a vaccine may be the most promising approach to prevent new infections, in many developing countries, experts say, screening with cheap tests-including visual inspection-continues to be the best hope for prevention and treatment.

TV Padma, New Delhi 